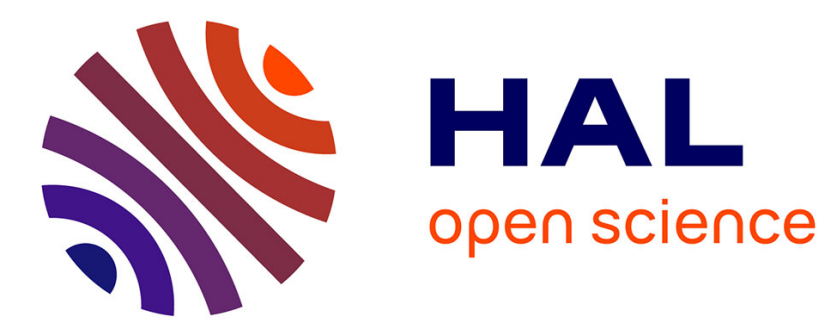

\title{
Technologies flexibles et collusion tacite
}

\author{
Armel Jacques
}

\section{To cite this version:}

Armel Jacques. Technologies flexibles et collusion tacite. Recherches Economiques de Louvain Louvain economic review, 2006, 72 (4), pp.385-413. 10.3917/rel.724.0385 . hal-01300695

\section{HAL Id: hal-01300695 \\ https://hal.univ-reunion.fr/hal-01300695}

Submitted on 15 Apr 2016

HAL is a multi-disciplinary open access archive for the deposit and dissemination of scientific research documents, whether they are published or not. The documents may come from teaching and research institutions in France or abroad, or from public or private research centers.
L'archive ouverte pluridisciplinaire HAL, est destinée au dépôt et à la diffusion de documents scientifiques de niveau recherche, publiés ou non, émanant des établissements d'enseignement et de recherche français ou étrangers, des laboratoires publics ou privés. 


\title{
Technologies flexibles et collusion tacite
}

\author{
Armel Jacques* \\ CERESUR, Université de La Réunion**
}

\section{$1 \quad$ Introduction}

Les années 1970 et 1980 ont vu l'apparition et le développement d'un nouveau type d'usine conçue autour de processus de production faisant une large place à l'informatique et à la robotique. Ces nouvelles technologies permettent une plus grande flexibilité de l'entreprise. Cette flexibilité se manifeste principalement dans deux dimensions: le rythme de production peut être facilement modifié (ce qui n'était pas le cas dans les usines «tayloriennes » de la période précédente) et la gamme de produits pouvant être fabriqués à partir des mêmes installations s'est considérablement accrue (voir Milgrom et Roberts, 1990, pour des exemples, et Caron, 1997, pour une perspective historique du développement de la production flexible). L'apparition de ces nouvelles technologies a profondément modifié l'organisation interne des firmes (Milgrom et Roberts, 1990). Elle a aussi des incidences sur les modalités de la concurrence dans les secteurs industriels. Malgré la parution d'un certain nombre de travaux, les conditions industrielles conduisant à l'adoption de technologies flexibles et les implications concurrentielles ne sont pas encore totalement connues. L'émergence des technologies flexibles intervient lorsque le coût fixe additionnel de cette technologie est faible par rapport à la taille du marché et lorsque les biens susceptibles d'être produits sont suffisamment différenciés (Röller et Tombak, 1990, et Kim,

* Je tiens à remercier Michel MOREAUX, Pierre PICARD, Saïd SOUAM et les deux rapporteurs de la revue pour leurs conseils et commentaires, qui ont permis d'améliorer ce travail. Les imperfections de ce texte me sont entièrement imputables.

** CERESUR, Université de La Réunion, Faculté de Droit et d'Économie, 15, avenue René Cassin, 97715 Saint-Denis messag cedex 9. Email : Armel.Jacques@univ-reunion.fr. 
Röller et Tombak, 1992). Le choix technologique des firmes dépend aussi fortement des possibilités d'observation des choix technologiques des firmes concurrentes (Boyer, Jacques et Moreaux, 1998). De meilleures possibilités d'observation renforcent l'attrait des technologies flexibles, car elles permettent de tirer pleinement partie de la valeur stratégique de cette flexibilité. Une plus grande concentration de l'industrie semble aussi favoriser l'adoption de technologies flexibles (Röller et Tombak, 1993). Le sens du lien de causalité est cependant ambigu. Enfin, une volatilité importante des goûts des consommateurs peut conduire une firme à adopter une technologie permettant de modifier facilement le design de ses produits (Chang, 1993 et 1998, Jacques, 2002). Les conséquences en matière de politique de la concurrence, mises en lumière par l'économie industrielle, peuvent être regroupées sous deux grands thèmes: les technologies flexibles rendent la concurrence plus vive, que la concurrence soit de type Cournot (Röller et Tombak, 1990) ou de type Bertrand (Norman et Thisse, 1999), lorsque le nombre de firmes est exogène; mais elles entraînent aussi une plus forte concentration (Chang, 1993, Eaton et Schmitt, 1994, Norman et Thisse, 1999). Cette concentration s'explique par la prolifération des produits que les technologies FMS (Flexible Manufacturing System) rendent possible, ce qui diminue le nombre des «niches » disponibles pour des entrants potentiels; mais surtout les technologies FMS, en multipliant le nombre des produits offerts, accroissent les possibilités des firmes de discriminer par les prix. Dans un modèle à la Hotelling, l'introduction de la discrimination par les prix a pour effet de supprimer les externalités des fusions et de la formation de cartels qui limitent la concentration dans ce genre de modèle (Eaton et Schmitt, 1994) ${ }^{1}$.

Cette littérature appréhende plusieurs aspects importants des effets concurrentiels de l'émergence de ces nouvelles technologies. Elle n'a cependant pas abordé le problème de la collusion tacite, pourtant essentiel. Cette étude se propose de combler, au moins partiellement, cette lacune : expliciter les interactions entre le choix technologique et les possibilités de collusion tacite. On sait que, dans un contexte de jeux répétés, un équilibre de collusion tacite peut émerger si les firmes se mettent d'accord sur un ensemble de règles de conduite spécifiant les prix et les quantités de chacune des parties à l'accord, ainsi que les mesures de rétorsion qui seraient prises si au moins l'une des firmes ne respectait pas le pacte de collusion. Ce type d'entente étant illégal, le non respect de l'accord ne peut pas être sanctionné par une cour de justice. Pour que l'accord soit respecté, les mesures de rétorsion prévues, augmentation des volumes produits et diminution des prix, doivent être telles que leur exécution entraînerait, pour la firme ayant violé l'accord, une diminution de ses profits actualisés supérieure au gain maximum pouvant être obtenu en s'écartant momentanément de ce qu'il était convenu qu'elle fasse. Les technologies flexibles, en rendant la concurrence plus intense lorsque les firmes se comportent de façon non coopérative, augmentent les possibilités de rétorsions, mais elles augmentent aussi les gains

1 Pour une synthèse plus détaillée et incluant d'autres aspects de la flexibilité voir Jacques (2003). 
pouvant être obtenus en violant l'accord tacite. Leur effet sur l'émergence possible d'un accord de collusion est donc a priori ambigu. Que cet effet soit positif ou négatif, les firmes le prendront en compte lors de leur choix technologique. La prise en compte des possibilités de collusion affecte donc les configurations technologiques choisies. Ces effets, a priori dans les deux sens, rendent particulièrement intéressante l'étude des interactions entre choix technologiques et collusion tacite ${ }^{2}$.

Pour étudier les incidences des choix technologiques sur les possibilités de collusion, on reprend la structure de base du modèle de Röller et Tombak (1990). Le modèle (présenté à la section 2) comprend deux phases. Lors de la première phase, les firmes choisissent simultanément une technologie. Deux types de technologies sont disponibles, une technologie flexible et des technologies « dédiées ». Ces choix sont non coopératifs et irréversibles. Lors de la deuxième phase, les firmes se livrent une concurrence infiniment répétée. On suppose que les firmes se font une concurrence en prix et choisissent simultanément la quantité maximale qu'elles acceptent de vendre à ce prix. On envisage aussi le cas où la concurrence est en quantités à la Cournot ${ }^{3}$. Un équilibre de collusion tacite peut émerger au cours de cette seconde phase. On suppose que les accords de collusion sont des équilibres à seuil du genre de ceux analysés dans l'étude pionnière de Friedman (1971). On compare, dans la section 3, les possibilités de collusion existant entre deux firmes ayant des technologies dédiées et celles existant entre deux firmes dotées de technologies flexibles. On montre que les technologies flexibles facilitent la collusion, lorsque la concurrence est en prix, mais que ce résultat est inversé si la concurrence est en quantités. La section 4 décrit les accords de collusion passés entre deux firmes ayant des technologies différentes. Dans la section 5 , on étudie les choix technologiques de la première phase du jeu. On montre que la possibilité de faire de la collusion dans la seconde phase du jeu favorise l'adoption des technologies flexibles. Cet effet est dû à la règle de partage adoptée pour sélectionner un équilibre de collusion unique entre deux firmes asymétriques. Les firmes choisissent la flexibilité afin d'être en position de force lors de la négociation établissant le partage des profits. On montre aussi que l'investissement supplémentaire en flexibilité consenti par les firmes n'est pas toujours compensé par les gains engendrés par la collusion tacite. Les firmes pourraient parfois réaliser des profits plus élevés si la collusion n'était pas possible.

2 Bourgeon et Smith (1998) étudient aussi l'impact des choix technologiques des firmes sur la collusion tacite. Leur travail est cependant très différent. II se situe dans la lignée de celui de Green et Porter (1984). Le choix technologique est un arbitrage entre le niveau du coût marginal et le coût fixe. Il est, en outre, renouvelé au début de chaque période. Les firmes, en choisissant un coût marginal élevé, diminuent leurs incitations à dévier. Lorsque le prix est bas, la probabilité que la cause en soit la faiblesse de la demande augmente, ce qui diminue le prix seuil au dessous duquel une guerre de prix est déclenchée. Le choix de technologies inefficientes en terme de coût de production permet donc de limiter la fréquence des guerres de prix et d'augmenter l'espérance de profit des firmes.

3 Dans le modèle de Röller et Tombak (1990), cette seconde étape n'était jouée qu'une fois et seule la concurrence à la Cournot était étudiée. 


\section{$2 \quad$ Le modèle}

Le modèle utilisé s'inspire fortement du modèle de duopole avec deux biens différenciés (A et B) présenté par Röller et Tombak (1990), la seconde étape de leur jeu étant ici répétée indéfiniment et la concurrence étant en prix, avec la possibilité pour les firmes de limiter les quantités vendues à ce prix.

Le jeu se décompose en deux phases. Lors de la première, les deux firmes choisissent simultanément une technologie. Deux types de technologies sont disponibles : une technologie flexible (notée FMS), ayant un coût fixe $F_{F M S} \geqslant 0$, qui permet de produire les deux biens, et des technologies dédiées (notées $\mathrm{DE}$ ), ayant un coût fixe $F_{D E} \geqslant 0$, qui ne permettent de produire qu'un seul des deux biens. On suppose : $2 F_{D E} \geqslant F_{F M S} \geqslant F_{D E}$. Le coût marginal de production, $c$, est constant et identique pour les deux technologies et pour les deux biens. On suppose que si la firme 1 [respectivement, la firme 2] choisit une technologie DE, elle choisit celle permettant de produire le bien A [respectivement, le bien B]. Les choix technologiques sont non-coopératifs et irréversibles. Ces choix ayant été effectués, ils deviennent connaissance commune.

Les firmes s'engagent, ensuite, dans un jeu de concurrence en prix indéfiniment répété. A chaque période, les firmes choisissent simultanément un prix pour chacun des types de bien qu'elles produisent ainsi qu'un quota indiquant la quantité maximale de ce bien qu'elles acceptent de vendre à ce prix. Elles ne produisent, cependant, que les quantités effectivement demandées. C'est dans cette seconde phase du jeu qu'un accord de collusion tacite peut apparaître ${ }^{4}$. On introduit des quotas pour permettre aux firmes, lorsqu'elles font de la collusion, de se répartir la demande sans aucune contrainte, en dehors de celles qui sont imposées par les technologies choisies. L'introduction de cette seconde variable stratégique ne modifie pas la nature de la concurrence en prix. Il en irait différemment si ces quantités maximales étaient choisies et observées avant que les firmes ne fixent leur prix (Kreps et Scheinkman, 1983) ou si l'une des firmes choisissait ces deux variables avant sa concurrente (Boyer et Moreaux, 1986). Les accords de collusion tacite ne peuvent comprendre ni des transferts de fonds ni des échanges de produits ${ }^{5}$ entre les deux firmes. Le partage des profits stipulé par un accord de collusion est déterminé par une règle inspirée de la solution du jeu coopératif de marchandage proposée par Nash $(1950)^{6}$. Lorsque les firmes sont symétriques, i.e. lorsqu'elles ont choisi la même technologie, cette négociation aboutit à un partage égalitaire ${ }^{7}$.

La demande est supposée identique à chaque période. Les deux biens sont des biens substituts non durables, et il n'y a pas d'habitude de con-

4 On étudie donc des accords de semi-collusion, c'est-à-dire ne portant que sur la seconde phase du jeu.

5 On pourrait imaginer des accords prévoyant des échanges de biens entre deux entreprises ayant des technologies dédiées ce qui leur permettrait de se comporter comme des entreprises flexibles.

6 La règle adoptée est définie explicitement au début de la section 4.

7 Ce n'est pas un résultat mais l'un des axiomes posés par Nash (1950). 
sommation ni d'effet d'accoutumance. Les fonctions de demande inverse sont données par :

$$
p^{X}\left(Q^{X}, Q^{Y}\right)=\max \left\{\alpha-Q^{X}-\lambda Q^{Y}, 0\right\} \quad X, Y \in\{A, B\} \text { et } X \neq Y
$$

où $\lambda \in[0,1]$ est un paramètre mesurant le degré de différenciation entre les deux biens.

Les fonctions de demande de l'agent représentatif qui en découlent sont :

$Q^{X}\left(p^{X}, p^{Y}\right)=$

$$
\left(\begin{array}{cc}
\frac{\alpha}{1+\lambda}-\frac{1}{1-\lambda^{2}} p^{X}+\frac{\lambda}{1-\lambda^{2}} p^{Y}, & \text { si } p^{X}<\alpha(1-\lambda)+\lambda p^{Y}, \\
\alpha-p^{X}, & \text { si } p^{X}<\alpha \text { et } p^{Y} \geqslant \alpha(1-\lambda)+\lambda p^{X}, \\
0 & \text { si } p^{X} \geqslant \alpha \text { et } p^{Y} \geqslant \alpha
\end{array}\right)
$$

Le facteur d'actualisation est égal à $\delta$. La première période de production intervient une période après le paiement du coût fixe.

\section{$3 \quad$ La flexibilité peut faciliter la collusion}

On étudie d'abord les possibilités de collusion dans chacune des configurations technologiques possibles, ces configurations étant, à cette étape $\mathrm{du}$ travail et jusqu'à la section 5 , exogènes. On en déduit l'impact de la configuration technologique sur la collusion tacite. On montre que pour certains niveaux d'actualisation, la collusion parfaite ${ }^{8}$ est soutenable lorsque les deux firmes sont flexibles mais n'est pas soutenable lorsque les deux firmes sont dédiées. Contrairement au cadre statique, où la flexibilité des firmes rend la concurrence plus vive, dans un cadre dynamique, la flexibilité peut atténuer la concurrence entre les firmes. On étudie, d'abord, la possibilité de collusion lorsque les deux firmes sont équipées de technologies dédiées. On étudie, ensuite, la soutenabilité de la collusion lorsque les deux firmes sont flexibles. On compare, enfin, les résultats obtenus dans ces deux situations.

\subsection{Les deux firmes ont des technologies dédiées}

Afin de déterminer pour quelles valeurs du facteur d'actualisation la collusion parfaite peut être soutenue, il est nécessaire de calculer les profits des firmes dans trois situations : lorsqu'elles respectent l'accord (collusion), lorsque les deux firmes se comportent de manière non coopérative (punition) et,

8 On appelle collusion parfaite un accord de collusion dans lequel les firmes choisissent le prix de monopole et collusion partielle un accord où le prix retenu est supérieur à celui de l'équilibre de Nash du jeu non répété mais inférieur au prix de monopole. 
enfin, lorsque l'une des firmes rompt l'accord alors que l'autre firme continue de le respecter (déviation).

Les deux premières situations sont assez simples à caractériser dans ce contexte où les deux firmes sont symétriques. S'il y a collusion tacite, les firmes maximisent les profits joints et se partagent le marché. La situation prévalant lors d'une éventuelle punition est l'équilibre de Nash du jeu non répété. Le tableau ci-dessous résume les caractéristiques de ces deux situations $^{9}$ :

\begin{tabular}{|l|l|l|}
\hline & Collusion & Punition \\
\hline Prix $\left(p_{1}^{A}=p_{2}^{B}\right)$ & $\alpha-\frac{1}{2}(\alpha-c)$ & $\frac{1-\lambda}{2-\lambda} \alpha+\frac{1}{2-\lambda} c$ \\
\hline Quantités $\left(q_{1}^{A}=q_{2}^{B}\right)$ & $\frac{1}{2(1+\lambda)}(\alpha-c)$ & $\frac{1}{(1+\lambda)(2-\lambda)}(\alpha-c)$ \\
\hline Quotas $\left(\bar{q}_{1}^{A}=\bar{q}_{2}^{B}\right)$ & $\frac{1}{2(1+\lambda)}(\alpha-c)$ & $\frac{2}{(1+\lambda)(2-\lambda)}(\alpha-c)$ \\
\hline Profit $\left(\pi_{1}=\pi_{2}\right)$ & $\frac{1}{4(1+\lambda)}(\alpha-c)^{2}=\pi^{c}$ & $\frac{1-\lambda}{(1+\lambda)(2-\lambda)^{2}}(\alpha-c)^{2}=\pi^{b n}$ \\
\hline
\end{tabular}

Sur le sentier de collusion, les firmes choisissent des quotas au moins égaux à la moitié de la production d'un monopole. Elles peuvent choisir des quotas plus élevés et laisser les consommateurs choisir aléatoirement la firme à laquelle ils souhaitent acheter. Si les consommateurs sont suffisamment nombreux et si leurs choix sont indépendamment distribués, on aboutit à des parts de marché égales pour les deux firmes. Sur le sentier de punition, les firmes doivent choisir des quotas supérieurs à leur production. Si les firmes choisissaient des quotas égaux à leur production, une firme pourrait augmenter son profit en augmentant son prix, car les consommateurs seraient alors dans l'impossibilité de se tourner vers l'autre firme. Si les firmes fixent des quotas suffisamment élevés ${ }^{10}$, les résultats obtenus sur les sentiers de collusion et de punition sont identiques à ceux trouvés en considérant une concurrence à la Bertrand classique (i.e. sans quotas). Les firmes ne peuvent pas augmenter leur profit en fixant des quotas plus faibles, étant donnée la stratégie jouée par leur concurrente ${ }^{11}$.

Il reste à caractériser la déviation optimale. La forme de celle-ci dépend du degré de substituabilité entre les deux produits. La baisse de prix que doit consentir une firme dédiée pour attirer tous les consommateurs est une fonction croissante du degré de substituabilité des deux biens. Si ce dernier est faible (inférieur à $\sqrt{3}-1$ ), la firme qui dévie peut s'emparer de la totalité du marché en ne baissant que faiblement son prix. En revanche, si les deux biens sont plus différenciés alors la baisse de prix qui doit être

9 Les exposants indiquent le bien considéré, les indices désignent la firme.

10 Dans le tableau, on a retenu le double de la quantité produite de façon arbitraire. Ce qui est important, c'est que les consommateurs ne soient pas rationnés.

11 Si les quotas étaient choisis et observés avant que les firmes ne choisissent les prix alors les firmes pourraient réduire la concurrence en prix en fixant des quotas plus faibles. Ici, les deux variables sont choisies simultanément, l'effet précédent est donc absent. 
consentie pour s'emparer de tout le marché est plus élevée et la firme qui dévie a alors intérêt à ne pas totalement exclure la firme rivale du marché.

\begin{tabular}{|l|l|l|}
\hline & Déviation si $\lambda<\sqrt{3}-1$ & Déviation si $\lambda \geqslant \sqrt{3}-1$ \\
\hline Prix $\left(p^{d}\right)$ & $\frac{\alpha+c}{2}-\frac{\lambda}{4}(\alpha-c)$ & $\frac{1}{2 \lambda}[(2 \lambda-1) \alpha+c]$ \\
\hline Quantités $\left(q^{d}\right)$ & $\frac{1}{1-\lambda^{2}} \frac{2-\lambda}{4}(\alpha-c)$ & $\frac{1}{2 \lambda}(\alpha-c)$ \\
\hline Profit & $\frac{1}{1-\lambda^{2}} \frac{(2-\lambda)^{2}}{16}(\alpha-c)^{2}=\pi^{d 2}$ & $\frac{2 \lambda-1}{4 \lambda^{2}}(\alpha-c)^{2}=\pi^{d 1}$ \\
\hline
\end{tabular}

La firme qui dévie fixe des quotas de production au moins égaux au niveau de production indiqués dans le tableau ci-dessus.

On peut maintenant calculer le facteur d'actualisation minimum, noté $\widehat{\delta}(\lambda)$, permettant de soutenir la collusion en fonction du degré de substituabilité des produits. On sait (Cf. Friedman, 1971) que si :

$$
\delta \geqslant \hat{\delta}(\lambda)=\frac{\pi^{d j}-\pi^{c}}{\pi^{d j}-\pi^{b n}}
$$

alors la stratégie à seuil est un équilibre parfait du jeu infiniment répété.

Si $1 \geqslant \lambda \geqslant \sqrt{3}-1$ :

$$
\hat{\delta}(\lambda)=\frac{\pi^{d 1}-\pi^{c}}{\pi^{d 1}-\pi^{b n}}=\frac{\left(\lambda^{2}+\lambda-1\right)(2-\lambda)^{2}}{(2 \lambda-1)(1+\lambda)(2-\lambda)^{2}-4 \lambda^{2}(1-\lambda)}
$$

Si $\sqrt{3}-1>\lambda>0$ :

$$
\hat{\delta}(\lambda)=\frac{\pi^{d 2}-\pi^{c}}{\pi^{d 2}-\pi^{b n}}=\frac{(2-\lambda)^{2}}{\lambda^{2}-8 \lambda+8}
$$

Si $\lambda=0$, alors les deux marchés sont indépendants. Chaque firme se comporte comme un monopole sur son marché quoi que fasse l'autre firme sur l'autre marché. Un accord de collusion est alors sans objet.

La fonction $\hat{\delta}(\lambda)$ est d'abord croissante puis décroissante. Sa valeur est égale à 0,5 lorsque $\lambda$ tend vers 0 et lorsque $\lambda$ est égal à 1 . Ce taux est donc toujours supérieur ou égal à 0,5 (à l'exception du cas $\lambda=0$ ).

\subsection{Les deux firmes ont des technologies flexibles}

On étudie, maintenant, le cas opposé où les deux firmes sont dotées de technologies FMS. Cette situation est très simple. S'il y a collusion tacite, les firmes (symétriques) maximisent les profits joints, en choisissant le prix de monopole, et se partagent les marchés de façon égalitaire. En cas de punition, les firmes jouent l'équilibre de Nash non-coopératif. Comme les 
firmes sont présentes sur les deux marchés, elles se livrent une concurrence avec des biens homogènes. Le prix d'équilibre est donc égal au coût marginal de production et les profits sont nuls. En cas de déviation, la firme qui dévie diminue ses deux prix d'un montant très faible et elle s'empare de la totalité du profit de monopole. On obtient (Les prix sont identiques sur les deux marchés. La quantité indiquée est produite par chacune des firmes sur chacun des marchés dans les deux premières situations et par la firme qui dévie dans la dernière) :

\begin{tabular}{|l|l|l|l|}
\hline & Collusion & Punition & Déviation \\
\hline Prix & $\alpha-\frac{1}{2}(\alpha-c)$ & $c$ & $\alpha-\frac{1}{2}(\alpha-c)-\varepsilon$ \\
\hline Quantités & $\frac{1}{4(1+\lambda)}(\alpha-c)$ & $\frac{1}{2(1+\lambda)}(\alpha-c)$ & $\frac{1}{2(1+\lambda)}(\alpha-c)$ \\
\hline Quotas & $\frac{1}{4(1+\lambda)}(\alpha-c)$ & $\frac{2}{2(1+\lambda)}(\alpha-c)$ & $\frac{1}{2(1+\lambda)}(\alpha-c)$ \\
\hline Profit & $\frac{1}{4(1+\lambda)}(\alpha-c)^{2}=\pi^{c}$ & $0=\pi^{b n}$ & $\frac{1}{2(1+\lambda)}(\alpha-c)^{2}=\pi^{d}$ \\
\hline
\end{tabular}

On peut maintenant calculer le facteur d'actualisation minimum permettant de soutenir la collusion parfaite en fonction du degré de substituabilité des produits. La stratégie à seuil est un équilibre parfait du jeu infiniment répété, si :

$$
\delta \geqslant \delta(\lambda)=\frac{\pi^{d}-\pi^{c}}{\pi^{d}-\pi^{b n}}=\frac{1}{2}
$$

La possibilité de soutenir la collusion parfaite est indépendante du degré de substituabilité des biens. Le facteur d'actualisation minimum permettant de soutenir la collusion parfaite entre deux firmes flexibles est égal au facteur d'actualisation minimum permettant à deux firmes monoproduits, produisant un bien homogène et se livrant une concurrence en prix, de soutenir un équilibre de collusion parfaite. Ce résultat rappelle celui établi par Bernheim et Whinston (1990); lorsque les marchés sont identiques, les contacts entre deux firmes sur plusieurs marchés indépendants ne permettent pas d'accroître les possibilités de collusion. Ce résultat, démontré pour des marchés indépendants, se généralise au cas de deux marchés reliés.

\subsection{Comparaison}

En comparant les facteurs d'actualisation minimum obtenus dans les deux cas précédents, on peut conclure ainsi :

Proposition 1 Si la variable stratégique est le prix alors la collusion parfaite est plus facile à soutenir entre deux firmes flexibles qu'entre deux firmes dédiées pour tous les niveaux de substituabilité entre les biens à l'exception $d u$ cas limite où les deux marchés sont parfaitement indépendants $(\lambda=0)$. 
La configuration technologique a donc un impact sur les possibilités de collusion. Les technologies flexibles ont deux effets. En permettant d'élargir la gamme des biens produits par chacune des firmes, elles diminuent la différenciation des produits entre les firmes et rendent la concurrence plus vive lorsque celles-ci se comportent de manière non-coopérative. Ce premier effet rend la punition potentielle plus dure en cas de déviation de l'accord de collusion tacite et donc facilite la possibilité de soutenir ce dernier. Mais, il y a un deuxième effet, qui va en sens opposé. La flexibilité permet à chacune des firmes d'augmenter le nombre de biens qu'elle produit. Lors de la phase de déviation, cela permet à la firme de s'emparer de la totalité des marchés tout en ne consentant qu'une diminution marginale de ses prix. Ce second effet rend la collusion plus délicate à mettre en oeuvre. Les calculs montrent cependant que le premier effet l'emporte pour tous les degrés de substituabilité des produits à l'exception du cas extrême où les deux marchés sont indépendants. L'émergence des technologies flexibles peut donc faciliter l'apparition de nouveaux accords de collusion tacite.

\subsubsection{Limites du résultat obtenu}

Il convient cependant d'apporter quelques nuances, qui limitent la portée du résultat obtenu. Premièrement, si la collusion est plus facile avec des technologies flexibles lorsque le facteur d'actualisation est élevé, ce résultat ne se généralise pas aux cas où le facteur d'actualisation est faible. Deuxièmement, si l'on considère que les firmes se font concurrence en quantités, et non plus en prix, le résultat est inversé : les technologies flexibles rendent la collusion parfaite plus difficile à soutenir.

Facteurs d'actualisation faibles et collusion partielle : On a montré que, lorsque les deux firmes sont équipées de technologies flexibles, la collusion parfaite est soutenable si et seulement si le facteur d'actualisation est supérieur ou égal à 0,5 . Si le facteur d'actualisation est plus faible alors non seulement la collusion parfaite n'est plus soutenable mais aucun degré de collusion partielle ne l'est non plus. En effet, quel que soit le profit de collusion partielle, le profit de déviation est nécessairement le double, lorsque les deux firmes sont flexibles, et donc, cet accord n'est pas soutenable pour un facteur d'actualisation inférieur à 0,5 . Il en résulte que, lorsque les deux firmes ont une technologie flexible, soit elles peuvent soutenir un équilibre de collusion parfaite, soit elles ne peuvent pas faire du tout de collusion.

Les choses sont plus complexes lorsque les deux firmes ont des technologies dédiées. Dans cette configuration technologique, lorsque le facteur d'actualisation est inférieur à $\widehat{\delta}(\lambda)$, la collusion parfaite ne peut plus être soutenue, mais des prix supérieurs à ceux de l'équilibre de Nash du jeu non répété sont encore soutenables. Les firmes peuvent donc encore soutenir des équilibres de collusion partielle. 
Pour des facteurs d'actualisation faibles, les technologies dédiées permettent donc de soutenir des équilibres de collusion partielle alors que les technologies flexibles ne le permettent pas.

Concurrence à la Cournot : Le résultat obtenu pour la concurrence en prix est inversé si la concurrence est en quantités à la Cournot. Pour expliquer le passage à une concurrence à la Cournot, on peut, dans le modèle précédent, modifier le timing des décisions et décider que les choix des quotas de production interviennent avant le choix des prix et sont observables par les firmes concurrentes.

Si la concurrence est en quantités, la collusion parfaite est soutenable entre deux firmes équipées de technologies dédiées si et seulement $\mathrm{si}^{12}$ :

$$
\delta \geqslant \frac{(2+\lambda)^{2}}{\lambda^{2}+8 \lambda+8}
$$

Ce seuil limite est une fonction croissante sur l'intervalle ]0, 1]. Il tend vers 0,5 lorsque $\lambda$ tend vers 0 et il est égal à $\frac{9}{17}$ lorsque les deux biens sont parfaitement homogènes. La collusion est donc d'autant plus facile à soutenir que les biens sont plus différenciés.

Si la concurrence est en quantités, la collusion parfaite est soutenable entre deux firmes équipées de technologies flexibles si et seulement si :

$$
\delta \geqslant \frac{9}{17}
$$

En comparant ce seuil avec celui obtenu lorsque les deux firmes ont des technologies DE, il apparaît:

Proposition 2 Si la variable stratégique est la quantité alors la collusion parfaite est plus facile à soutenir entre deux firmes dédiées qu'entre deux firmes flexibles pour tous les niveaux de substituabilité entre les biens.

Ce résultat est l'opposé de celui obtenu lorsque la concurrence est en prix. Dans les deux types de concurrence, les technologies flexibles augmentent les possibilités de punition mais elles augmentent aussi les possibilités de déviation. Lorsque la concurrence est en prix, c'est le premier effet qui domine. Lorsque la concurrence est en quantités, le second effet l'emporte. L'impact de la configuration technologique sur les possibilités de collusion entre les firmes dépend donc de façon cruciale du type de concurrence.

12 Pour rendre la lecture moins fastidieuse, les développements techniques concernant la concurrence en quantités ont été renvoyés en annexe. 


\section{$4 \quad$ Les structures industrielles mixtes}

On s'intéresse, maintenant, à la configuration industrielle mixte où l'une des firmes est équipée d'une technologie flexible tandis que l'autre firme a une technologie dédiée.

Ce dernier cas soulève un problème méthodologique particulièrement complexe. En effet, on sait que, dans un jeu répété, lorsque le facteur d'actualisation est élevé, il existe une multitude d'équilibres de Nash parfaits (Folk theorem). Le problème est alors d'en sélectionner un. Lorsque les firmes sont symétriques, il est assez naturel de sélectionner un équilibre qui attribue des parts de marchés égales aux deux firmes. Lorsque les firmes sont asymétriques, la désignation d'un «point focal » est nettement moins évidente. Les réponses à ce problème varient beaucoup dans la littérature existante ${ }^{13}$.

Dans cette étude, le processus de sélection retenu s'inspire de la règle de partage de Nash (1950). Afin de pouvoir comparer les résultats avec ceux de la section précédente, on se limite à l'ensemble des équilibres de collusion parfaite (appelé H), c'est à dire ceux pour lesquels les prix fixés sur les deux marchés sont des prix de monopole. Parmi ces équilibres, on choisit celui qui maximise la fonction $\left(\pi_{1}-\pi_{1}^{b n}\right)\left(\pi_{2}-\pi_{2}^{b n}\right)$. La possibilité qu'ont les firmes de fixer des quotas de production leur permet de mettre en oeuvre l'équilibre choisi ${ }^{14}$. Sur le sentier de collusion, les quotas fixés sont, donc, exactement égaux aux quantités vendues. Les consommateurs ne sont pas rationnés stricto sensu, mais ils peuvent être obligés d'acheter le produit de l'une des firmes après l'épuisement de la quantité maximale que l'autre firme est prête à vendre. Cependant, comme ces produits sont homogènes et vendus au même prix, il n'y a pas d'impact sur le niveau d'utilité obtenu par les consommateurs. On utilise, donc, la règle de partage de Nash, en prenant comme point de menace l'équilibre de Nash du jeu constitutif non répété et en restreignant l'ensemble des couples possibles aux équilibres de collusion parfaite.

Sans perte de généralité, on pose que la firme ayant une technologie FMS est la firme 1, et que la technologie DE de la firme 2 permet de produire le bien B. On note $\pi^{m}$ le profit d'une firme en situation de monopole sur les deux marchés.

13 Les critères de sélection retenus dans la littérature sont très divers : Davidson et Deneckere (1990) supposent que les firmes se partagent le marché proportionnellement à leurs capacités. Pénard (1997) considère que, si les capacités sont suffisantes pour servir le marché, le partage du marché ne dépend pas des capacités des firmes. Bae (1987) fait l'hypothèse que les firmes se partagent le marché de manière telle que leurs incitations à tricher soient égales. D'autres études proposent d'appliquer une axiomatique de marchandage. Osborne et Pitchik $(1983,1987)$ appliquent la règle proposée par Nash (1950). Cette règle est aussi retenue par Schmalensee (1987), Harrington $(1989,1991)$ et Jéhiel (1992).

14 C'est d'ailleurs la raison de leur introduction dans le modèle. 
La solution retenue est telle que :

$$
\begin{aligned}
& \left(\pi_{1}^{c}, \pi_{2}^{c}\right) \in \underset{H}{\arg \max }\left(\pi_{1}-\pi_{1}^{b n}\right)\left(\pi_{2}-\pi_{2}^{b n}\right) \\
& s / c \quad \delta \geqslant \frac{\pi_{i}^{d}-\pi_{i}^{c}}{\pi_{i}^{d}-\pi_{i}^{b n}} \quad i=1,2 \\
& \text { et } \pi^{m}=\pi_{1}+\pi_{2}
\end{aligned}
$$

La résolution de ce programme conduit aux résultats suivants (voir annexe pour les détails). Si le facteur d'actualisation est supérieur ou égal à 0,5 , le partage des profits entre les deux firmes est donné par la règle de Nash (1950) et les contraintes d'incitation à ne pas dévier ne sont pas saturées. On a alors :

$$
\pi_{1}^{c}=\frac{3-\lambda}{8(1+\lambda)}(\alpha-c)^{2} \quad \text { et } \quad \pi_{2}^{c}=\frac{1}{8}(\alpha-c)^{2}
$$

Si le facteur d'actualisation est plus faible, l'accord précédent ne peut plus être soutenu, car la contrainte d'incitation à ne pas dévier de la firme ayant une technologie FMS est violée. Cependant, pour certaines valeurs du facteur d'actualisation, la contrainte d'incitation de la firme ayant une technologie DE n'est pas saturée. Il est donc possible d'obtenir un accord de collusion parfaite soutenable en réallouant une partie du quota de la firme DE à la firme FMS pour toutes les valeurs de $\delta$ supérieures à $\tilde{\delta}(\lambda)$. Où $\tilde{\delta}(\lambda)$ est défini par :

$$
\tilde{\delta}(\lambda)= \begin{cases}\frac{(2-\lambda)^{2}}{8-4 \lambda-3 \lambda^{2}} & \text { si } \lambda \leqslant \sqrt{3}-1 \\ \frac{2 \lambda-1}{\lambda^{2}+2 \lambda-1} & \text { si } \lambda>\sqrt{3}-1\end{cases}
$$

Dans cette zone $\left(\frac{1}{2} \geqslant \delta \geqslant \tilde{\delta}(\lambda)\right)$, l'accord tacite de collusion répartit les profits de la manière suivante:

$$
\begin{aligned}
& \pi_{1}^{c}=\pi^{m}-\pi_{2}^{c}=\frac{1}{4(1+\lambda)}[2-\delta(1+\lambda)](\alpha-c)^{2} \\
& \pi_{2}^{c}=\delta\left(\pi^{m}-\pi_{1}^{b n}\right)=\frac{\delta}{4}(\alpha-c)^{2}
\end{aligned}
$$

Le facteur d'actualisation minimum $\tilde{\delta}(\lambda)$ pour que cet accord soit soutenable est égal à 0,5 pour $\lambda=0$, il décroit ensuite avec $\lambda$, jusqu'à $\lambda=0,5$, il est alors approximativement égal à 0,4286 . Puis il croit avec $\lambda$ pour atteindre la valeur de 0,5 lorsque $\lambda=1$. On a donc $: \tilde{\delta}(\lambda) \leqslant \widehat{\delta}(\lambda)$. 
En comparant ce seuil à ceux obtenus précédemment, on obtient le résultat suivant :

Proposition 3 Si la variable stratégique est le prix, la collusion parfaite est plus facile à soutenir entre deux firmes ayant des technologies différentes qu'entre deux firmes ayant la même technologie pour tous les niveaux de substituabilité entre les biens (à l'exception du cas limite où les deux marchés sont parfaitement indépendants $(\lambda=0))$.

Ce résultat est un peu surprenant car la littérature sur la collusion avance souvent que la collusion est plus facile à soutenir lorsque les firmes sont symétriques (Pénard, 1997). La difficulté rencontrée par les firmes pour soutenir un équilibre de collusion parfaite tient, cependant, souvent à la règle de partage du marché adoptée. Par exemple, Pénard (1997) suppose, dans un modèle de duopole, que si chaque firme a une capacité suffisante pour servir la moitié du marché, alors le marché est partagé de façon égalitaire entre les firmes. Cet équilibre est difficilement soutenable, lorsque les capacités des firmes sont très asymétriques. Car, dans ce cas, une seule firme a réellement les capacités nécessaires pour dissuader une déviation de sa rivale. La firme ayant la capacité la plus importante est incitée à dévier, car l'autre firme ne peut pas la punir durement. Dans le modèle de Pénard (1997), les firmes ne disposent que d'un seul instrument, le choix du prix; elles ne peuvent pas limiter leurs ventes à un niveau inférieur à leur capacité. La firme ayant la capacité la plus faible ne peut donc pas abandonner une partie de ses parts de marché à la firme ayant la capacité la plus élevée afin de l'inciter à respecter l'accord. Compte, Jenny et Rey (2002), au contraire, permettent aux firmes de fixer des parts de marché de tailles différentes et dépendant des capacités des firmes. Cette possibilité accroît les possibilités de collusion tacite. Les auteurs montrent que si les capacités totales de l'industrie sont faibles alors les possibilités de collusion sont maximales lorsque les capacités totales sont reparties de façon égalitaire entre les firmes. Cette répartition est celle qui maximise la punition potentielle contre la firme la plus susceptible de dévier (qui est celle ayant la capacité la plus élevée). En revanche, lorsque les capacités des autres firmes sont suffisantes pour ramener le prix à zéro en cas de punition, alors, pour maximiser les possibilités de collusion, il faut attribuer toutes les capacités supplémentaires à la firme la plus grande. Les attribuer à une autre firme augmente son incitation à dévier sans augmenter les possibilités de punition dans l'industrie.

Dans le modèle étudié ici, lorsqu'une des firmes passe d'une technologie DE à une technologie FMS, cela augmente les possibilités de punition de l'industrie, mais parallèlement, cela augmente aussi les possibilités de déviation. Le premier effet l'emporte, lorsque l'autre firme a une technologie DE; tandis que le second effet domine, lorsque l'autre firme a une technologie FMS. On retrouve des effets analogues à ceux de Compte, Jenny et Rey (2002). Lorsque les deux firmes ont des technologies DE, les possibilités de punition sont faibles et on peut augmenter les possibilités de collusion en renforçant la punition. Lorsque l'une des firmes est équipée d'une techno- 
logie FMS, les possibilités de collusion sont déjà importantes. Il est alors plus important de limiter les capacités de déviations des firmes que de renforcer les possibilités de collusion ${ }^{15}$. Si, dans le modèle considéré dans cette étude, on supprime la possibilité pour les firmes de fixer des quotas alors la collusion parfaite devient très difficile à soutenir lorsque les firmes ont des technologies différentes. Soit les deux firmes fixent le prix de monopole sur le marché B et elles obtiennent chacune une part de marché égale à $50 \%$, soit la firme 1 fixe un prix un peu supérieur et la firme 2 obtient tout le marché du bien B. Dans les deux cas, il faut que le facteur d'actualisation soit relativement élevé pour que ces situations soient des équilibres de Nash parfaits. La différence entre le résultat obtenu ici et celui de Pénard (1997) semble donc surtout dû à la différence des critères de sélection de l'équilibre retenus.

Si la concurrence est en quantités, on obtient le résultat suivant ${ }^{16}$ :

Proposition 4 Si la variable stratégique est la quantité alors les possibilités de collusion parfaite sont les mêmes lorsque les firmes ont des technologies différentes que lorsqu'elles sont équipées de technologies flexibles et inférieures à celles existant lorsque les firmes sont équipées de technologies DE.

Dans ce cas non plus, l'asymétrie technologique entre les firmes ne rend pas nécessairement la collusion parfaite plus difficile à soutenir. Ces résultats suggèrent que les problèmes éventuels causés par une asymétrie technologique entre les firmes sont des problèmes de coordination sur un même équilibre beaucoup plus que des problèmes de soutenabilité de ces équilibres.

\section{Choix technologiques}

On dispose, maintenant, de tous les éléments pour décrire les choix technologiques des firmes lors de la phase préliminaire du jeu. Pour éviter les problèmes dus à l'existence éventuelle d'équilibres de collusion partielle, on se restreint à étudier les cas où le facteur d'actualisation est suffisamment élevé pour que la collusion parfaite soit soutenable dans toutes les configurations technologiques possibles. On pose donc $\delta \geqslant \hat{\delta}(\lambda)$. Lors de la première période, les firmes choisissent donc simultanément leur technologie avant de jouer l'équilibre de collusion parfaite correspondant à la configuration technologique d'équilibre qui émerge. Ces choix technologiques sont irréversibles.

Avant de caractériser les configurations technologiques d'équilibre lorsque la collusion tacite est possible, on va décrire les configurations tech-

15 On obtient aussi une relation non monotone entre les capacités de production d'une industrie et les possibilités de soutenir un équilibre de collusion parfaite dans le modèle de Brock et Scheinkman (1985).

16 Voir l'annexe pour les détails techniques. 
nologiques d'équilibre en l'absence de collusion. Lorsque la variable stratégique est le prix ${ }^{17}$, le choix de la technologie FMS par une des firmes entraîne des profits nuls pour l'autre firme (hors coût fixe). La seconde firme n'entre donc pas sur le marché à moins que $F_{D E}=0$, ce qui entraîne que $F_{F M S}=0$ car on a supposé $F_{F M S} \leqslant 2 F_{D E}$. Les situations où l'une des firmes entre sur le marché et choisit la technologie FMS et l'autre firme n'entre pas sont donc des équilibres du jeu sans collusion tacite. Si $F_{D E}$ est suffisament faible, il existe un autre équilibre, dans lequel les deux firmes entrent sur le marché et choisissent des technologies dédiées à des produits différents.

On va supposer qu'en l'absence de collusion, c'est ce dernier équilibre qui apparaîtrait. La comparaison des équilibres dans les deux situations (avec et sans collusion) est plus claire si le nombre de firmes actives reste constant. On peut justifier le choix de cet équilibre de deux façons. Premièrement, on peut supposer que les deux firmes sont déjà actives sur le marché avec des technologies DE, lorsque apparaît une nouvelle technologie flexible. Le choix technologique des firmes est alors un choix d'adoption de la nouvelle technologie. Deuxièmement, on peut aussi éliminer le problème en séparant la première période du jeu en deux étapes. Lors de la première, les firmes décident d'entrer ou non sur le marché. Ce choix devient connaissance commune. Lors de la seconde étape, les firmes choisissent une technologie. En l'absence de collusion, à l'équilibre (unique), les deux firmes entrent sur le marché et choisissent ensuite des technologies dédiées.

On calcule les fonctions de meilleure réponse des firmes au choix technologique de leur concurrente, lorsque la collusion tacite est possible. On caractérise ensuite les technologies choisies à l'équilibre. On montre enfin qu'il est possible que les profits des firmes lorsque la collusion tacite est possible soient plus faibles que lorsqu'elle est exclue.

\subsection{Fonctions de meilleure réponse}

Le facteur d'actualisation est suffisamment élevé pour que la collusion soit soutenable quelle que soit la configuration technologique choisie. La somme des profits des deux firmes est la même dans chacune des configurations technologiques. En revanche la distribution de ces profits entre les firmes est affectée par les choix technologiques. Le choix technologique d'une firme dépend donc d'un arbitrage entre deux éléments. La technologie flexible a un coût fixe plus élevé mais cette technologie augmente le pouvoir de négociation de la firme et lui permet d'obtenir, lors de la négociation, une part des profits plus importante.

17 Les équilibres obtenus, en l'absence de collusion, lorsque la concurrence est en quantités, sont donnés par Röller et Tombak (1990) et Boyer, Jacques et Moreaux (1998). Lorsque le coût fixe de la technologie FMS est faible par rapport à la taille du marché et que le degré de substituabilité des biens est faible, la configuration technologique d'équilibre est (FMS,FMS). Dans les cas opposés, à l'équilibre, on a (DE,DE). Dans les cas intermédiaires, le jeu admet deux équilibres en stratégies pures (FMS,FMS) et (DE,DE). 


\subsubsection{Meilleure réponse technologique à une technologie DE}

La technologie FMS est la meilleure réponse à la technologie DE si et seulement si :

$$
\begin{gathered}
\sum_{i=1}^{\infty} \delta^{i} \frac{3-\lambda}{8(1+\lambda)}(\alpha-c)^{2}-F_{F M S} \geqslant \sum_{i=1}^{\infty} \delta^{i} \frac{1}{4(1+\lambda)}(\alpha-c)^{2}-F_{D E} \\
\Leftrightarrow \frac{\delta}{1-\delta} \frac{1-\lambda}{8(1+\lambda)}(\alpha-c)^{2} \geqslant F_{F M S}-F_{D E}
\end{gathered}
$$

On note $\delta^{f}(\lambda)$ le facteur d'actualisation au dessus duquel cette condition est vérifiée.

\subsubsection{Meilleure réponse technologique à une technologie FMS}

La technologie FMS est la meilleure réponse à la technologie FMS si et seulement si :

$$
\begin{gathered}
\sum_{i=1}^{\infty} \delta^{i} \frac{1}{4(1+\lambda)}(\alpha-c)^{2}-F_{F M S} \geqslant \sum_{i=1}^{\infty} \delta^{i} \frac{1}{8}(\alpha-c)^{2}-F_{D E} \\
\Leftrightarrow \frac{\delta}{1-\delta} \frac{1-\lambda}{8(1+\lambda)}(\alpha-c)^{2} \geqslant F_{F M S}-F_{D E}
\end{gathered}
$$

Cette fonction de meilleure réponse technologique est identique à la précédente. En effet, pour ces valeurs du facteur d'actualisation, la configuration technologique affecte seulement la distribution des profits entre les deux firmes. Donc, FMS est la meilleure réponse à DE, si la part supplémentaire de profit que permet de capter cette technologie est supérieure au coût additionnel de la flexibilité. Cependant, dans ce cas, l'autre firme en choisissant aussi FMS « récupère » cette part des profits. Cette stratégie est optimale car la part de profit en jeu est supérieure au coût additionnel de la flexibilité. Donc FMS est la meilleure réponse à FMS.

\section{2 Équilibres technologiques}

De la caractérisation des fonctions de meilleures réponses, on déduit les configurations technologiques d'équilibre. Les résultats sont présentés à la figure 1 (les valeurs des paramètres sont fixées à $F_{F M S}-F_{D E}=5$ et $\alpha-c=10)$.

Lorsque le facteur d'actualisation est supérieur à $\hat{\delta}(\lambda)$, la collusion est possible dans toutes les configurations technologiques. Dans cette zone, la configuration technologique n'a d'effet que sur la répartition des profits entre les deux firmes. La flexibilité n'est donc choisie que pour renforcer le 


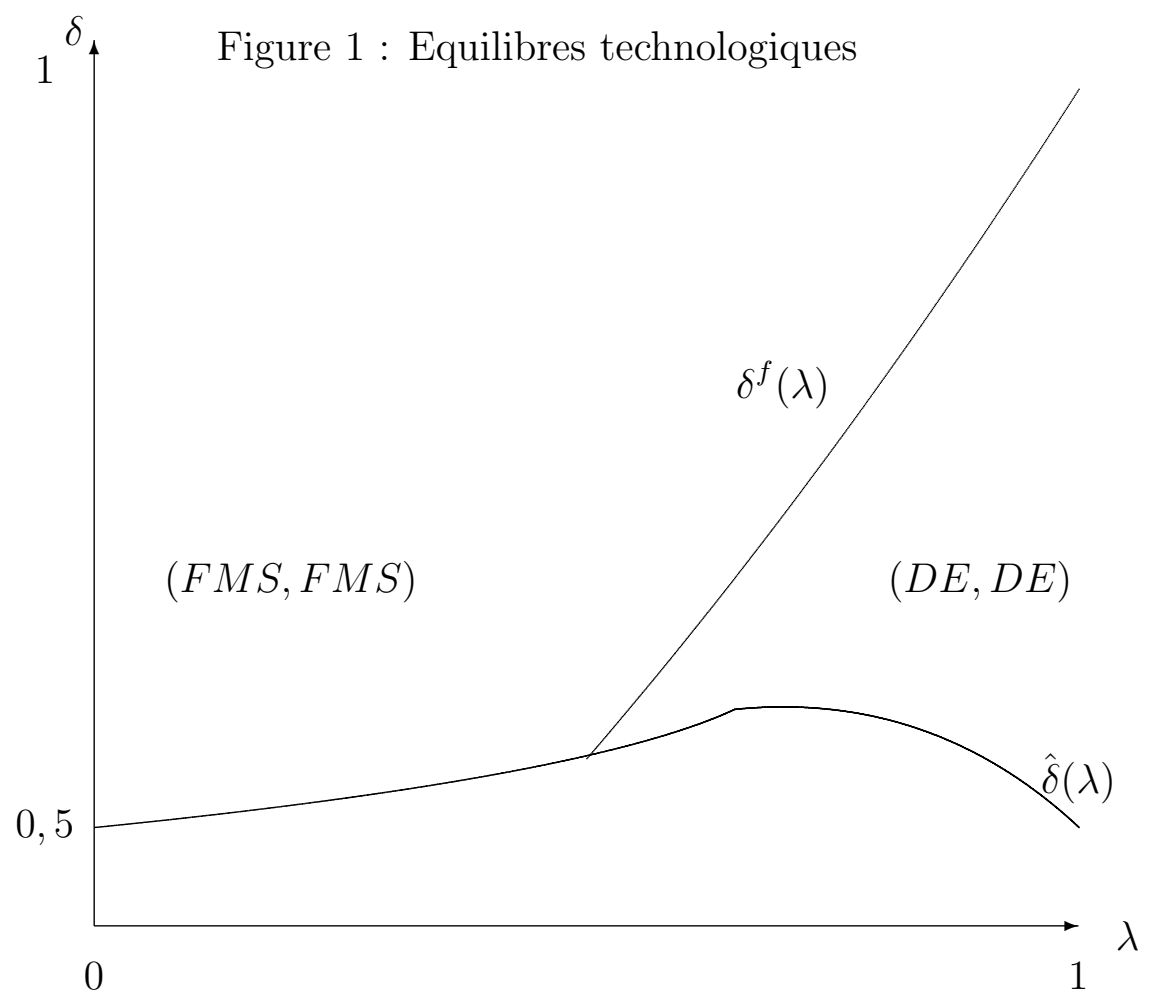

pouvoir de négociation de la firme lors de l'allocation des quotas de production. Les firmes choisissent la flexibilité si le coût fixe additionnel de cette technologie est inférieur à la part supplémentaire de profit qu'elle permet de capter. La part de profit supplémentaire est une fonction décroissante du degré de substituabilité entre les deux biens. En effet, plus les biens sont différenciés et plus les profits de monopole sont élevés. En outre, cette part de profit est d'autant plus valorisée par la firme que le facteur d'actualisation est élevé. L'équilibre technologique est donc (FMS, FMS) lorsque $\delta$ est élevé et $\lambda$ est faible et l'équilibre technologique est (DE, DE) lorsque $\delta$ est plus faible et $\lambda$ est plus élevé. Comme on l'a noté lors du calcul des fonctions de meilleure réponse technologique, dans cette zone une configuration technologique mixte n'est jamais un équilibre de Nash (à l'exception des points composant la frontière).

On peut aussi remarquer que la collusion tacite encourage l'adoption de technologies flexibles. En effet, on observe à l'équilibre des zones où la configuration technologique est (FMS, FMS), alors que sans collusion cette configuration ne peut pas exister. En l'absence de collusion, à l'équilibre, soit les deux firmes ont des technologies dédiées, soit une seule des deux firmes est présente sur le marché. La collusion tacite est donc un facteur favorable à l'adoption de technologies plus flexibles. 


\subsection{Les accords de semi-collusion peuvent diminuer le profit des firmes}

La possibilité pour les firmes de passer des accords de collusion tacite dans la seconde phase du jeu peut les inciter à investir dans une technologie flexible ce qu'elles n'auraient pas fait si la collusion n'était pas possible. Ce choix technologique entraîne un coût fixe additionnel. Ce coût peut-il être supérieur au gain engendré par la collusion? Pour avoir cet effet, il faut que trois conditions soient réunies :

- Les firmes doivent choisir des technologies flexibles lorsque la collusion tacite est possible. Cette condition est équivalente à la technologie FMS est la meilleure réponse à la technologie FMS.

- Elles doivent choisir des technologies dédiées lorsque la collusion tacite n'est pas possible (pour des raisons exogènes indépendantes des possibilités de soutenir l'accord de collusion). Cette seconde condition, équivalente à la technologie DE est la meilleure réponse à la technologie DE en l'absence de possibilité de collusion tacite, est toujours vérifiée si les deux firmes sont présentes sur le marché.

- Les profits d'une industrie (DE,DE) sans collusion doivent être supérieurs à ceux d'une industrie (FMS,FMS) dans laquelle un accord de collusion tacite existe. Formellement, on doit avoir :

$$
\begin{gathered}
\sum_{i=1}^{\infty} \delta^{i} \frac{1-\lambda}{(1+\lambda)(2-\lambda)^{2}}(\alpha-c)^{2}-F_{D E} \geqslant \sum_{i=1}^{\infty} \delta^{i} \frac{1}{4(1+\lambda)}(\alpha-c)^{2}-F_{F M S} \\
\Leftrightarrow F_{F M S}-F_{D E} \geqslant \frac{\delta}{1-\delta} \frac{\lambda^{2}}{4(1+\lambda)(2-\lambda)^{2}}(\alpha-c)^{2}
\end{gathered}
$$

On note $\delta^{*}(\lambda)$ le facteur d'actualisation au dessous duquel cette condition est vérifiée.

La figure 2 (les valeurs des paramètres sont identiques à celle de la figure précédente) montre qu'il existe une zone où ces trois conditions sont remplies. La possibilité de faire de la collusion tacite peut donc diminuer le profit des firmes.

Ce résultat peut sembler surprenant, il est pourtant assez fréquent dans les modèles de semi-collusion (Matsui (1989), Fershtman et Gandal (1994) et Brod et Shivakumar (1999)). La collusion tacite crée des rentes supplémentaires. Les firmes sont donc prêtes à fournir des efforts supplémentaires pour s'approprier ces rentes et les efforts consentis peuvent être plus grands que les rentes finalement obtenues. Brod et Shivakumar (1999) ont proposé un modèle où les firmes choisissent de façon non-coopérative un niveau de R\&D pour diminuer leur coût de production, et donnant lieu à des externalités pour l'autre firme, avant de passer un accord de collusion. Ils montrent que, selon les valeurs des paramètres, le profit des firmes et le surplus des consommateurs peuvent augmenter ou diminuer lorsque la collusion est possible. 


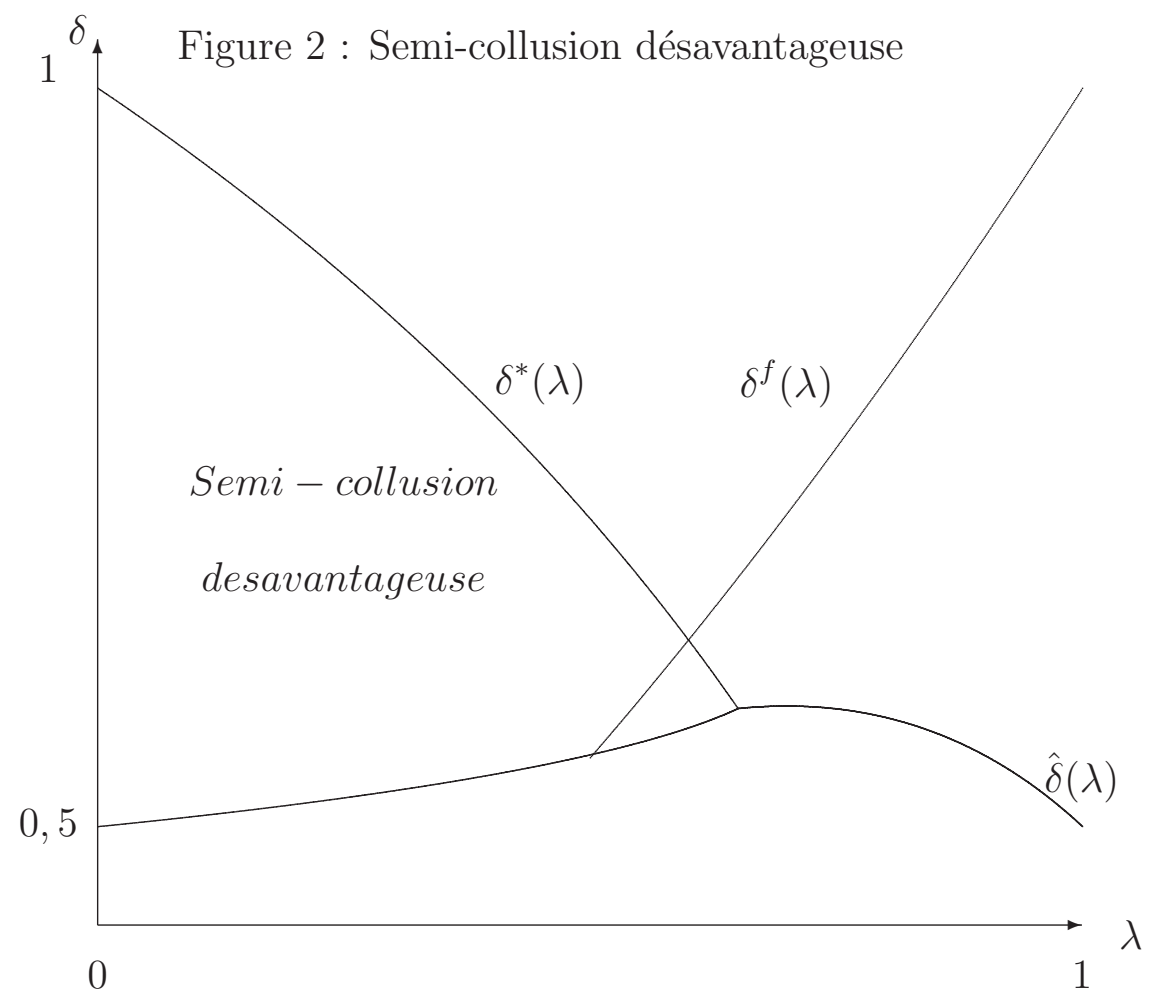

Dans le modèle de cette étude, les investissements supplémentaires diminuent clairement le bien-être social. Ils représentent un coût pour les firmes et ils n'ont aucun effet bénéfique pour les consommateurs. Ils ne permettent pas de baisser les prix, ni d'augmenter la variété des produits.

Les firmes peuvent donc avoir intérêt à s'engager avant le début du jeu à coopérer avec la puissance publique pour rendre plus difficiles les accords de collusion tacite, par exemple en adoptant des systèmes de comptabilité faisant clairement apparaître leur coût de production pour faciliter le contrôle des autorités publiques. Le problème est que, une fois le choix technologique effectué, les firmes ont intérêt à revenir sur, leur engagement et à tenter de mettre en oeuvre un accord de collusion.

\subsection{Concurrence à la Cournot}

Contrairement aux deux sections précédentes où les résultats obtenus dans le cas de la concurrence en quantités étaient différents de ceux obtenus dans le cas de la concurrence en prix, les résultats obtenus dans cette section sont très semblables. Lorsque le facteur d'actualisation est suffisamment élevé pour que la collusion parfaite soit soutenable dans les trois configurations 
technologiques possibles, les fonctions de meilleures réponses technologiques sont les mêmes que dans le cas de la concurrence en prix. Ceci est dû au fait que, dans le cas asymétrique, la différence des profits réalisés par les deux firmes à l'équilibre de Nash du jeu non répété lorsque la variable stratégique est la quantité est identique à celle obtenue lorsque la variable stratégique est le prix $^{18}$. Il en découle que les choix technologiques d'équilibre sont les mêmes. En outre, les configurations technologiques obtenues à l'équilibre avec collusion sont plus flexibles que celles obtenues sans collusion. Donc, comme dans le cas de la concurrence en prix, la collusion encourage l'adoption de technologies flexibles. Enfin, comme dans le cas précédent, la collusion tacite, en incitant les firmes à investir dans une technologie plus coûteuse, peut entraîner une diminution du profit des firmes.

\section{Conclusion}

Cette étude a permis d'établir qu'il existe des interactions entre les choix technologiques de flexibilité et la collusion tacite. Ces interactions dépendent, cependant, du mode de concurrence entre les firmes. Lorsque la concurrence est en prix, la soutenabilité de la collusion tacite est une fonction non monotone du degré de flexibilité de l'industrie. Les possibilités de collusion tacite sont maximales lorsque l'une des firmes a une technologie flexible et l'autre firme est équipée d'une technologie dédiée. Elles sont minimales lorsque les deux firmes ont des technologies dédiées. Dans des modèles statiques, l'adoption des technologies flexibles augmente la concurrence entre les firmes (Röller et Tombak, 1990, Norman et Thisse, 1999). Ce résultat ne se généralise pas aux modèles dynamiques. Lorsque la concurrence est en quantités, en revanche, l'adoption de technologies flexibles rend la collusion tacite plus difficile à soutenir. Ces résultats ont, cependant, été obtenus sous des hypothèses particulières et il reste à tester leurs robustesses pour d'autres fonctions de demande, un plus grand nombre de firmes ou des stratégies de collusion différentes, de type «bâton et carotte» (Abreu, 1986).

Quel que soit le mode de concurrence entre les firmes, la perspective de pouvoir mettre en oeuvre un équilibre de collusion tacite incite les firmes à choisir des technologies flexibles. Ces dernières augmentent le pouvoir de négociation des firmes lors de l'allocation des parts de marché entre les firmes. Les technologies flexibles devraient donc apparaître plus fréquemment dans les industries dont les caractéristiques facilitent la collusion tacite. Röller et Tombak (1993) ont constaté qu'empiriquement, la flexibilité des firmes était plus importante dans les industries plus concentrées. Cette constatation est cohérente avec les résultats obtenus dans cette étude. L'émergence d'équilibres de collusion tacite n'augmente, cependant, pas nécessairement les profits des firmes. Les recettes plus importantes générées par la collu-

18 Voir annexe. 
sion incite les firmes à augmenter leurs investissements en première période et l'augmentation des investissements peut, pour certaines valeurs des paramètres du modèle, être plus importante que l'augmentation des recettes et peut donc entraîner une diminution du profit des firmes. 


\section{Annexe A : configuration technologique mixte et concur- rence en prix}

On doit résoudre le problème suivant:

$$
\begin{aligned}
& \left(\pi_{1}^{c}, \pi_{2}^{c}\right) \in \underset{H}{\arg \max }\left(\pi_{1}-\pi_{1}^{b n}\right)\left(\pi_{2}-\pi_{2}^{b n}\right) \\
& s / c \quad \delta \geqslant \frac{\pi_{i}^{d}-\pi_{i}^{c}}{\pi_{i}^{d}-\pi_{i}^{b n}} \quad i=1,2 \\
& \text { et } \pi^{m}=\pi_{1}+\pi_{2}
\end{aligned}
$$

Dans un premier temps, on résoud ce problème sans prendre en compte les contraintes de non déviation. On cherche alors l'ensemble des facteurs d'actualisation pour lesquels l'équilibre calculé est un équilibre de Nash parfait. On étudie ensuite les cas où au moins l'une des contraintes est saturée.

En négligeant la contrainte de non déviation, on obtient:

$$
\pi_{1}^{c}=\frac{1}{2}\left(\pi^{m}+\pi_{1}^{b n}-\pi_{2}^{b n}\right) \text { et } \pi_{2}^{c}=\frac{1}{2}\left(\pi^{m}-\pi_{1}^{b n}+\pi_{2}^{b n}\right)
$$

Pour avoir une expression explicite de ces profits de collusion, on calcule l'équilibre de Nash du jeu constitutif non répété. Sur le marché B, les deux firmes se livrent une concurrence en prix $^{19}$. Le prix d'équilibre sur ce marché est donc égal au coût marginal de production et on a trivialement $\pi_{2}^{b n}=0$. La firme 1 choisit sur le marché A le prix qui maximise son profit, compte tenu du fait que $p^{B}=c$. On en déduit :

$$
\pi_{1}^{b n}=\frac{1-\lambda}{4(1+\lambda)}(\alpha-c)^{2}
$$

On obtient alors :

$$
\pi_{1}^{c}=\frac{3-\lambda}{8(1+\lambda)}(\alpha-c)^{2} \quad \text { et } \quad \pi_{2}^{c}=\frac{1}{8}(\alpha-c)^{2}
$$

On cherche maintenant à préciser l'ensemble des facteurs d'actualisation pour lesquels cette situation est un équilibre de Nash parfait. Les profits de déviation s'obtiennent sans difficulté. Si la firme 1 dévie, elle le fait en diminuant son prix sur le marché B d'un montant infime. Elle s'empare alors de tout le profit de monopole, $\pi_{1}^{d}=\pi^{m}$. Si la firme 2 dévie, sa déviation optimale est identique à la déviation optimale d'une firme DE

19 A l'équilibre, les firmes choisissent des quotas de production suffisamment élevés pour que, quels que soient les prix choisis par la firme concurrente, les consommateurs qui s'adressent à une firme ne soient pas rationnés. 
faisant face à une autre firme DE. En effet, en diminuant très légèrement son prix, elle s'empare de la totalité du marché B. Elle doit alors décider de la diminution de prix supplémentaire qu'elle est prête à consentir pour conquérir une partie ou la totalité de l'autre marché. Les profits permis par cette déviation ont été calculés dans la section précédente.

Ne pas dévier est une stratégie d'équilibre pour la firme FMS si et seulement si :

$$
\delta \geqslant \frac{\pi_{1}^{d}-\pi_{1}^{c}}{\pi_{1}^{d}-\pi_{1}^{b n}}=\frac{1}{2}
$$

Pour la firme DE ne pas dévier est une stratégie d'équilibre si et seulement si :

Premier cas : $\lambda \leqslant \sqrt{3}-1$.

$$
\delta \geqslant \frac{\pi_{2}^{d}-\pi_{2}^{c}}{\pi_{2}^{d}-\pi_{2}^{b n}}=\frac{2-4 \lambda+3 \lambda^{2}}{(2-\lambda)^{2}}
$$

Second cas : $\lambda \geqslant \sqrt{3}-1$.

$$
\delta \geqslant \frac{\pi_{2}^{d}-\pi_{2}^{c}}{\pi_{2}^{d}-\pi_{2}^{b n}}=\frac{4 \lambda-2-\lambda^{2}}{4 \lambda-2}
$$

Le facteur d'actualisation minimum pour que ne pas dévier soit la stratégie de la firme DE est égal à 0,5 pour $\lambda=0$, il décroit ensuite avec $\lambda$, jusqu'à $\lambda=0,5$, puis il croit avec $\lambda$ pour atteindre la valeur de 0,5 lorsque $\lambda=1$.

L'accord de collusion que l'on a caractérisé est donc un équilibre de Nash parfait pour tous les facteurs d'actualisation supérieurs à 0,5 . Pour les facteurs inférieurs à 0,5 , cet accord ne peut pas être soutenu. Cependant, dans certains cas, un accord de collusion reste possible. En effet la contrainte d'incitation à ne pas dévier de la firme FMS est violée mais la contrainte d'incitation de la firme DE n'est pas saturée. Il est donc possible d'obtenir un accord de collusion soutenable en réallouant une partie du quota de production de la firme DE à la firme FMS.

La contrainte de non déviation de la firme 1 est saturée, on a donc:

$$
\delta=\frac{\pi^{m}-\pi_{1}^{c}}{\pi^{m}-\pi_{1}^{b n}} \Rightarrow \delta\left(\pi^{m}-\pi_{1}^{b n}\right)=\pi^{m}-\pi_{1}^{c}=\pi_{2}^{c}
$$

Il reste à vérifier que la firme DE n'a pas intérêt non plus à dévier. Un accord de collusion est donc possible si et seulement si :

$$
\delta \geqslant \frac{\pi_{2}^{d}-\pi_{2}^{c}}{\pi_{2}^{d}}=\frac{\pi_{2}^{d}-\delta\left(\pi^{m}-\pi_{1}^{b n}\right)}{\pi_{2}^{d}}
$$


Premier cas : $\lambda \leqslant \sqrt{3}-1$.

$$
\delta \geqslant \frac{(2-\lambda)^{2}}{8-4 \lambda-3 \lambda^{2}}
$$

Second cas : $\lambda \geqslant \sqrt{3}-1$.

$$
\delta \geqslant \frac{2 \lambda-1}{\lambda^{2}+2 \lambda-1}
$$

Dans cette zone, l'accord tacite de collusion répartit les profits de la manière suivante :

$$
\begin{aligned}
& \pi_{1}^{c}=\pi^{m}-\pi_{2}^{c}=\frac{1}{4(1+\lambda)}[2-\delta(1+\lambda)](\alpha-c)^{2} \\
& \pi_{2}^{c}=\delta\left(\pi^{m}-\pi_{1}^{b n}\right)=\frac{\delta}{4}(\alpha-c)^{2}
\end{aligned}
$$

\section{Annexe B : concurrence à la Cournot}

\section{Configurations technologiques symétriques}

Les deux firmes ont des technologies dédiées

Lorsque les deux firmes ont des technologies dédiées, on a :

\begin{tabular}{|l|l|l|l|}
\hline & Collusion & Punition & Déviation \\
\hline Prix $\left(p^{A}=p^{B}\right)$ & $\alpha-\frac{1}{2}(\alpha-c)$ & $\alpha-\frac{1+\lambda}{2+\lambda}(\alpha-c)$ & $\alpha-\left(1+\frac{3}{2} \lambda\right) \frac{1}{2(1+\lambda)}(\alpha-c)$ \\
\hline Quantités $\left(q_{1}^{A}=q_{2}^{B}\right)$ & $\frac{1}{2(1+\lambda)}(\alpha-c)$ & $\frac{1}{2+\lambda}(\alpha-c)$ & $\frac{2+\lambda}{4(1+\lambda)}(\alpha-c)$ \\
\hline Profit & $\frac{1}{4(1+\lambda)}(\alpha-c)^{2}=\pi^{c}$ & $\frac{1}{(2+\lambda)^{2}}(\alpha-c)^{2}=\pi^{c n}$ & $\frac{(2+\lambda)^{2}}{16(1+\lambda)^{2}}(\alpha-c)^{2}=\pi^{d}$ \\
\hline
\end{tabular}

Les deux firmes ont des technologies flexibles

Définir l'équilibre de collusion dans ce cas nécessite la résolution d'une légère difficulté. Si la détermination du prix de collusion, des quantités produites et des profits des firmes ne pose aucune difficulté, la répartition de la production des firmes entre les marchés est moins immédiate. La solution la plus satisfaisante est de retenir la répartition qui permet de soutenir le plus facilement la collusion. Comme les profits de collusion et ceux de punition sont indépendant de cette répartition, la répartition qui permet le plus facilement la collusion est celle qui minimise le profit d'une firme qui dévie. On va montrer que la répartition optimale est un partage égalitaire des deux marchés. 
Sans perte de généralité, on suppose que la firme qui dévie est la firme 1.

Lorsqu'il y a collusion, les quotas de production attribués à la firme 2 sont tels que :

$$
q_{2}^{A}+q_{2}^{B}=\frac{1}{2(1+\lambda)}(\alpha-c)
$$

On note $\gamma$ la part de la production de la firme 2 vendue sur le marché A. Les quantités produites par la firme 1, si elle dévie, sont données par sa fonction de meilleure réponse. Après quelques calculs, on en déduit que ses quantités sont égales à :

$$
\begin{aligned}
q_{1}^{A} & =\frac{\alpha-c}{2(1+\lambda)}-\frac{1}{2} \frac{\alpha-c}{2(1+\lambda)} \gamma \\
q_{1}^{B} & =\frac{\alpha-c}{2(1+\lambda)}-\frac{1}{2} \frac{\alpha-c}{2(1+\lambda)}(1-\gamma)
\end{aligned}
$$

On peut calculer les prix lors de la phase de déviation :

$$
\begin{aligned}
& p^{A}=c+\frac{\alpha-c}{4(1+\lambda)}(2+\lambda-\gamma+\lambda \gamma) \\
& p^{B}=c+\frac{\alpha-c}{4(1+\lambda)}(1+2 \lambda+\gamma-\lambda \gamma)
\end{aligned}
$$

Le profit de la firme qui dévie est donc égal à :

$$
\pi^{d}(\gamma)=\frac{(\alpha-c)^{2}}{16(1+\lambda)^{2}}\left[5+4 \lambda-2(1-\lambda) \gamma+2(1-\lambda) \gamma^{2}\right]
$$

On a donc

$$
\frac{\partial \pi^{d}(\gamma)}{\partial \gamma}=\frac{(\alpha-c)^{2}}{16(1+\lambda)^{2}}[-2(1-\lambda)+4(1-\lambda) \gamma]
$$

Expression qui s'annule en $\gamma=\frac{1}{2}$. La dérivée seconde étant positive, cette valeur est celle qui minimise les profits de déviation donc qui maximisent les possibilités de collusion. Q.E.D.

Lorsque les deux firmes ont des technologies flexibles, on a donc:

\begin{tabular}{|l|l|l|l|}
\hline & Collusion & Punition & Déviation \\
\hline Prix $\left(p^{A}=p^{B}\right)$ & $\alpha-\frac{1}{2}(\alpha-c)$ & $\alpha-\frac{2}{3}(\alpha-c)$ & $\alpha-\frac{5}{8}(\alpha-c)$ \\
\hline Quantités $\left(q_{1}^{A}=q_{1}^{B}=q_{2}^{A}=q_{2}^{B}\right)$ & $\frac{1}{4(1+\lambda)}(\alpha-c)$ & $\frac{1}{3(1+\lambda)}(\alpha-c)$ & $\frac{3}{8(1+\lambda)}(\alpha-c)$ \\
\hline Profit & $\frac{1}{4(1+\lambda)}(\alpha-c)^{2}=\pi^{c}$ & $\frac{2}{9(1+\lambda)}(\alpha-c)^{2}=\pi^{c n}$ & $\frac{9}{32(1+\lambda)}(\alpha-c)^{2}=\pi^{d}$ \\
\hline
\end{tabular}




\section{Configurations technologiques asymétriques}

La solution du jeu de marchandage décrite par Nash est telle que :

$$
\begin{aligned}
& \left(\pi_{1}^{c}, \pi_{2}^{c}\right) \in \underset{H}{\arg \max }\left(\pi_{1}-\pi_{1}^{c n}\right)\left(\pi_{2}-\pi_{2}^{c n}\right) \\
& s / c \quad \delta \geqslant \frac{\pi_{i}^{d}-\pi_{i}^{c}}{\pi_{i}^{d}-\pi_{i}^{c n}} \quad i=1,2
\end{aligned}
$$

On procède comme pour la concurrence en prix. Dans un premier temps, on résoud le problème sans prendre en compte les contraintes de non déviation. On cherche alors l'ensemble des facteurs d'actualisation pour lesquels l'équilibre calculé est un équilibre de Nash parfait. On étudie ensuite les cas où au moins l'une des contraintes est saturée.

$$
\begin{array}{r}
\left(\pi_{1}^{c}, \pi_{2}^{c}\right) \in \underset{H}{\arg \max }\left(\pi_{1}-\pi_{1}^{c n}\right)\left(\pi_{2}-\pi_{2}^{c n}\right) \\
\Rightarrow\left(\begin{array}{l}
\pi_{1}^{c}=\frac{1}{2}\left(\pi^{m}+\pi_{1}^{c n}-\pi_{2}^{c n}\right) \\
\pi_{2}^{c}=\frac{1}{2}\left(\pi^{m}-\pi_{1}^{c n}+\pi_{2}^{c n}\right)
\end{array}\right)
\end{array}
$$

Pour avoir une expression explicite de ces profits de collusion, on calcule l'équilibre de Nash du jeu constitutif non répété. Dans ce cas, l'équilibre de Nash du jeu non répété est de la forme (Cf. Röller et Tombak, 1990; Boyer, Jacques et Moreaux, 1998) :

$$
\begin{array}{rlrl}
q_{1}^{A} & =\frac{1}{2(1+\lambda)}(\alpha-c) \quad q_{1}^{B}=\frac{2-\lambda}{2(1+\lambda)} \frac{\alpha-c}{3} \\
q_{2}^{A} & =0 \quad q_{2}^{B}=\frac{1}{3}(\alpha-c) \\
p^{A} & =\frac{3-\lambda}{6}(\alpha-c)+c \quad p^{B}=\frac{\alpha-c}{3}+c \\
\pi_{1}^{c n} & =\frac{13-5 \lambda}{36(1+\lambda)}(\alpha-c)^{2} & \pi_{2}^{c n}=\frac{1}{9}(\alpha-c)^{2}
\end{array}
$$

\section{Remarque}

$$
\pi_{1}^{c n}-\pi_{2}^{c n}=\frac{1-\lambda}{4(1+\lambda)}(\alpha-c)^{2}=\pi_{1}^{b n}-\pi_{2}^{b n}
$$

La différence des profits réalisés par les deux firmes à l'équilibre de Nash du jeu non répété lorsque la variable stratégique est la quantité est identique à celle obtenue lorsque la variable stratégique est le prix.

Cette remarque implique que les profits des firmes, lorsqu'il y a collusion, sont eux aussi identiques dans les deux situations. Donc

$$
\pi_{1}^{c}=\frac{3-\lambda}{8(1+\lambda)}(\alpha-c)^{2} \quad \text { et } \quad \pi_{2}^{c}=\frac{1}{8}(\alpha-c)^{2}
$$


De la valeur de ces profits, on déduit les quotas de production alloués à chacune des deux firmes :

$$
\begin{aligned}
& q_{1}^{A}=\frac{1}{2(1+\lambda)}(\alpha-c) \quad q_{1}^{B}=\frac{1-\lambda}{4(1+\lambda)}(\alpha-c) \\
& q_{2}^{A}=0 \quad q_{2}^{B}=\frac{1}{4}(\alpha-c)
\end{aligned}
$$

En introduisant ces quotas dans les fonctions de meilleure réponse des firmes, on peut calculer la déviation optimale pour chacune des deux firmes.

Déviation optimale de la firme 1:

$$
\begin{array}{rlrl}
q_{1}^{d A} & =\frac{1}{2(1+\lambda)}(\alpha-c) & q_{1}^{d B} & =\frac{3-\lambda}{8(1+\lambda)}(\alpha-c) \\
p^{d A} & =\frac{4-\lambda}{8}(\alpha-c)+c & p^{B}=\frac{3}{8}(\alpha-c)+c \\
\pi_{1}^{d} & =\frac{25-7 \lambda}{64(1+\lambda)}(\alpha-c)^{2} &
\end{array}
$$

Déviation optimale de la firme 2 :

$$
\begin{aligned}
q_{2}^{d A} & =0 \quad q_{2}^{d B}=\frac{3}{8}(\alpha-c) \\
p^{B} & =\frac{3}{8}(\alpha-c)+c \quad \pi_{2}^{d}=\frac{9}{64}(\alpha-c)^{2}
\end{aligned}
$$

On peut maintenant calculer l'ensemble des facteurs d'actualisation pour lesquels l'accord de collusion décrit ci-dessus est soutenable.

Ne pas dévier est une stratégie d'équilibre pour la firme 1 si et seulement si :

$$
\delta \geqslant \frac{\pi_{1}^{d}-\pi_{1}^{c}}{\pi_{1}^{d}-\pi_{1}^{C N}}=\frac{9}{17}
$$

Pour la firme DE ne pas dévier est une stratégie d'équilibre si et seulement si :

$$
\delta \geqslant \frac{\pi_{2}^{d}-\pi_{2}^{c}}{\pi_{2}^{d}-\pi_{2}^{C N}}=\frac{9}{17}
$$

Lorsque le facteur d'actualisation est supérieur à $\frac{9}{17}$ alors les deux contraintes d'incitation à ne pas tricher sont vérifiées, l'accord de collusion décrit ci-dessus est donc un équilibre de Nash parfait. Lorsque le facteur d'actualisation est égal à $\frac{9}{17}$ alors les deux contraintes sont saturées; on ne peut donc pas soutenir un accord de collusion en dessous de ce facteur d'actualisation. 


\section{Références}

Abreu D. (1986), "Extremal equilibria of oligopolistic supergames", Journal of Economic Theory, 39, pp. 191-225.

Bae H. (1987), "A price-setting supergame between two heterogeneous firms", European Economic Review, 31, pp. 1159-1171.

Bernheim B. et D. Whinston (1990), "Multimarket contact and collusive behavior", Rand Journal of Economics, 21, pp. 1-26.

Bourgeon J-M. et R. Smith, (1998), "Technology choice and tacit collusion", mimeo.

Boyer M., A. Jacques et M. Moreaux (1998), "Better observability promotes the adoption of more flexible technologies", mimeo, GREMAQ (Toulouse) et CIRANO (Montréal).

Boyer M. et M. Moreaux (1986), «Rationnement, anticipations rationnelles et équilibres de Stackelberg », Annales d'Économie et de Statistique, 1 , pp. 55-73.

Brock W. et J. Scheinkman (1985), "Price setting supergames with capacity constraints", Review of Economic Studies, 52, pp. 371-382.

Brod A. et R. Shivakumar (1999), "Advantageous semi-collusion", Journal of Industrial Economics, 47, pp. 221-230.

Caron F, (1997), Les deux révolutions industrielles du XX siècle, Paris, Albin Michel.

Chang M.H. (1993), "Flexible manufacturing, uncertain consumer tastes, and strategic entry deterrence", Journal of Industrial Economics, 41, pp. 77-90.

Chang M.H. (1998), "Product switching cost and strategic flexibility", Journal of Economics and Management Strategy, 7, pp. 461-488.

Compte O., F. Jenny et P. Rey (2002), "Capacity constraints, mergers and collusion", European Economic Review, 46, pp. 1-29.

Davidson C. et R. Deneckere, (1990), "Excess capacity and collusion", International Economic Review, 31, pp. 521-541.

Eaton C.B. et N. Schmitt, (1994), "Flexible manufacturing and market structure", American Economic Review, 84, pp. 875-888.

Fershtman C. et N. Gandal, (1994), "Disadvantageous semicollusion", International Journal of Industrial Organization, 12, pp. 141-154.

Friedman J.W, (1971), "A non-cooperative equilibrium for supergames", Review of Economic Studies, 28, pp. 1-12.

Green E. et R. Porter (1984), "Noncooperative collusion under imperfect price formation", Econometrica, 52, pp. 87-100.

Harrington J.E.Jr. (1989), "Collusion among asymmetric firms : the case of different discount factors", International Journal of Industrial Organization, 7, pp. 289-307. 
Harrington J.E.Jr. (1991), "The determination of price and output quotas in a heterogeneous cartel", International Economic Review, 32, pp. 767792.

Jacques A. (2002), "Product switching cost and strategic flexibility : correction", Journal of Economics and Management Strategy, 11, pp. 547549 .

Jacques A. (2003), «La flexibilité technologique: un survol de la littérature », Revue d'économie politique, 113, pp. 587-624.

Jehiel P. (1992), "Product differentiation and price collusion", International Journal of Industrial Organization, 10, pp. 633-641.

Kim T., L.H. Röller et M. Tombak (1992), "Strategic choice of flexible production technologies and welfare implications : corregidum and addendum", Journal of Industrial Economics, 40, pp. 233-235.

Kreps D. et J. Scheinkman (1983), "Quantity precommitment and Bertrand competition yield Cournot outcomes", Bell Journal of Economics, 14, pp. 326-337.

Matsui A. (1989), "Consumer-benefited cartels under strategic capitalinvestment competition", International Journal of Industrial Organization, 7, pp. 451-470.

Milgrom P. et J. Roberts (1990), "The economics of modern manufacturing : technology, strategy and organization", American Economic Review, 80, pp. 511-528.

Nash J.F.Jr. (1950), "The bargaining problem", Econometrica, 28, pp. 513518.

Norman G. et J-F. Thisse (1999), "Technology choice and market structure : strategic aspects of flexible manufacturing", Journal of Industrial Economics, 47, pp. 345-372.

Osborne M. et C. Pitchik (1983), "Profit-sharing in a collusive industry", European Economic Review, 22, pp. 59-74.

Osborne M. et C. Pitchik (1987), "Cartels, Profits and excess capacity", International Economic Review, 28, pp. 413-428.

Pénard T. (1997), « Choix de capacités et comportements stratégiques : une approche par la théorie des jeux répétés », Annales d'Économie et de Statistique, 46, pp. 203-224.

Röller L.H. et M. Tombak (1990), "Strategic choice of flexible production technologies and welfare implications", Journal of Industrial Economics, 38, pp. 417-431.

Röller L.H. et M. Tombak (1993), "Competition and investment in flexible technologies", Management Science, 39, pp. 107-114.

Schmalensee R. (1987), "Competitive advantage and collusive optima", International Journal of Industrial Organization, 5, pp. 351-367. 
